\title{
Stability and reachability analysis of a hybrid model of luminescence in the marine bacterium Vibrio fischeri
}

\author{
Calin Belta ${ }^{1}$, Jonathan Schug ${ }^{2}$, Thao Dang ${ }^{1}$ \\ Vijay Kumar ${ }^{1}$, George J. Pappas ${ }^{1}$, Harvey Rubin ${ }^{3}$, \\ ${ }^{1}$ GRASP Laboratory \\ ${ }^{2}$ Center for Bioinformatics \\ ${ }^{3}$ School of Medicine \\ University of Pennsylvania, Philadelphia, PA, USA \\ and \\ Paul Dunlap \\ Department of Molecular, Cellular and Developmental Biology \\ University of Michigan, Ann Arbor, MI, USA \\ calinegrasp.cis.upenn.edu
}

\begin{abstract}
This paper addresses the mathematical modeling and analysis of the quorum sensing system found in unicellular bacteria that exhibit bioluminescence. The luminescence is governed by the expression of genes in the cell, which in turn is controlled by the density of cells in a population. The paper illustrates the application of standard tools in control theory and some recent tools in hybrid systems to the quorum sensing system, and demonstrates that bioluminescence can be modeled and understood as the output of a switched dynamical system.
\end{abstract}

\section{Introduction}

Vibrio fischeri is a marine bacterium which can be found both as free-living organism and as a symbiont of some marine fish and squid. As a free-living organism, V. fisheri exists at low densities and appears to be non-luminescent. As a symbiont, the bacteria live at high densities and are, usually, luminescent.

The luminescence in $V$. fischeri is controlled by the transcriptional activation of the lux genes. The lux regulon [10] is organized in two transcriptional units, $O_{L}$ and $O_{R}$. See Figure 1. The leftward operon, $O_{L}$, contains the luxR gene encoding the protein ${ }^{1}$ LuxR, a transcriptional regulator of the system. The rightward operon $O_{R}$ contains seven genes luxICDABEG. The transcription of the luxI gene results in the production of protein LuxI. This protein is required for endogenous production of autoinducer, Ai, a small

\footnotetext{
${ }^{1}$ We use italics (e.g., luxR) to indicate the genes and plain font to denote the protein expressed by the gene (e.g., LuxR)
}

membrane-permeant signal molecule (acyl-homoserine lactone). The genes $\operatorname{lux} A$ and $\operatorname{lux} B$ code for the luciferase subunits, which in turn are responsible for luminescence. $\operatorname{lux} C$, lux $D$, and lux $E$ code for polypeptides of the fatty-acid reductase, which generates aldehyde substrate for luciferase. Along with LuxR and LuxI, cAMP receptor protein (CRP) plays an important role in controlling luminescence. The network of biochemical reactions in the cell is as follows [10, 11]: The autoinducer $A i$ binds to protein LuxR to form a complex Co which binds to the lux box shown in Figure 1. The lux box is in the middle of a regulatory region between the two transcriptional units (operons). This region also contains a binding site for CRP. The transcription from the luxR promoter is activated by the binding of CRP to its binding site, and the transcription of the luxICDABEG by the binding of Co to the lux box [4]. However, growth in the levels of $C o$ and cAMP/CRP inhibit $l u x R$ and luxICDABEG transcription, respectively. These feedback loops are shown through dotted arrows with '+' and '-' signs in Figure 1.

Our goal in this paper is to develop and analyze a model for the genetic regulation of luminescence in Vibrio fischeri $[4,10]$. Our approach to understanding the bacterial quorum-sensing system couples standard tools in control theory [6] with some recent tools that have been developed for hybrid systems [7, 8]. We will be interested mainly in studying the stability and reachability of possible equilibrium points. For example, one can ask if a single $V$. fischeri bacterium in the right environment can eventually reach a steady state of luminescence. In our work, this translates to the existence, stability and reachability of an equilibrium in a discrete mode of the hybrid system.

We focus on two forms of $V$. fischeri: an isolate from nature (wild type) with the native quorum-sensing system in- 
tact and a mutant strain of this species [4] that is defective in one of the key regulatory elements of the system. We first develop continuous and hybrid models for the biochemical reactions in the wild type in Section 2, and then simulate them to study the quorum sensing phenomenon in Section 3. The hybrid model lends itself to the computation of equilibrium points and the analytical study of stability of the equilibrium points in Section 4. The reachability analysis of a luminescent equilibrium in a mutant is developed in Section 5. Finallv. we formulate our conclusions.

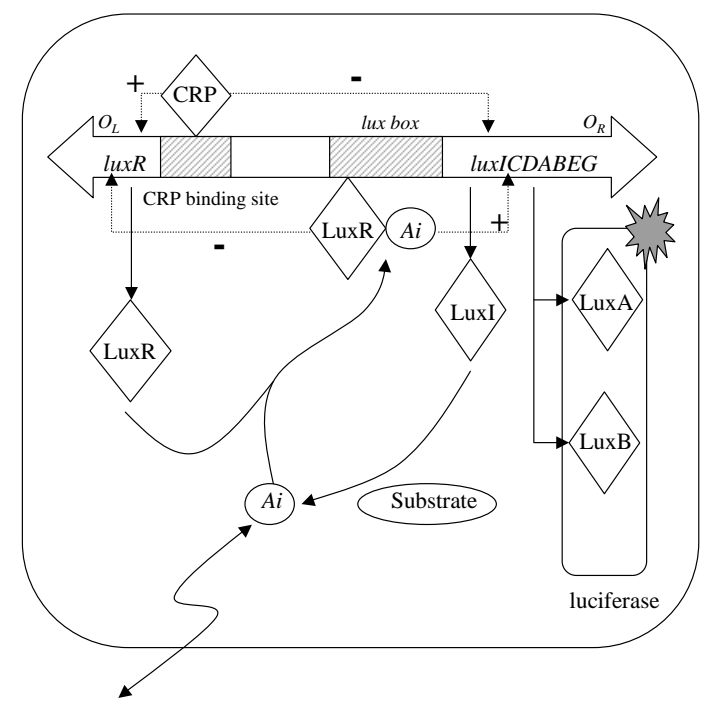

Figure 1: A portion of DNA showing the $\operatorname{luxR}$ and $\operatorname{luxICDABEG}$ genes and the binding sites for the complex LuxR-Ai and CRP.

\section{Mathematical models}

\subsection{Continuous model}

A typical gene has a single promoter, but multiple regulatory sites, both activating and inhibiting, distributed through a regulatory region. We model the regulation using functions found in the biology literature [5]:

$$
\begin{gathered}
\Phi\left(X, \kappa_{X m}, \nu_{X m}\right)=\frac{X^{\nu_{X}}}{\kappa_{X m}^{\nu_{X} m}+X^{\nu_{X} m}}, \\
\Psi\left(X, \kappa_{X m}, \nu_{X m}\right)=1-\Phi\left(X, \kappa_{X m}, \nu_{X m}\right),
\end{gathered}
$$

where $X$ is the concentration of some species with regulatory effect on the transcription of mRNA $m . \nu_{X m}$ is called the cooperativity coefficient and $\kappa_{X m}$ is the concentration of $X$ at which transcription of $m$ is "half-maximally" activated. The graph of function $\Phi$, the so called sigmoid, is given for illustration in Figure 2. To model the luminescence phenomenon in $V$. fischeri, we restrict our attention to a nine dimensional state space, each component (with the exception of the population $x_{0}$ ) denoting the appropriate nano-molar concentration. This model is derived from

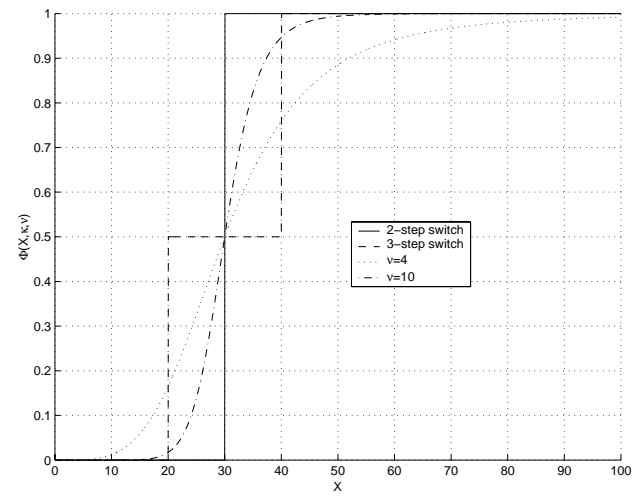

Figure 2: The function $\phi(X, \kappa, \nu)$ for $\kappa=30$ along with two examples of piecewise constant abstractions.

the observations documented in [4] and is described by:

$$
\begin{aligned}
\dot{x}_{0}= & k_{G} x_{0} \\
\dot{x}_{1}= & T_{c}\left(\Psi\left(x_{8}, \kappa_{\text {Co-icdabeg }}, \nu_{\text {Co-icdabeg }}\right)\right. \\
& \left.\Phi\left(c_{\mathrm{CRP}}, \kappa_{\mathrm{CRPr}}, \nu_{\mathrm{CRPr}}\right)+b\right)-\frac{x_{1}}{H_{\mathrm{RNA}}}-k_{G} x_{1} \\
\dot{x}_{2}= & T_{c}\left(\Phi\left(x_{8}, \kappa_{\mathrm{Co}-\mathrm{icdabeg}}, \nu_{\mathrm{Co}-\mathrm{icdabeg}}\right)\right. \\
& \left.\Psi\left(c_{\mathrm{CRP}}, \kappa_{\mathrm{CRPr}}, \nu_{\mathrm{CRPr}}\right)+b\right)-\frac{x_{2}}{H_{\mathrm{RNA}}}-k_{G} x_{2} \\
\dot{x}_{3}= & T_{l} x_{1}-x_{3} / H_{\mathrm{sp}}-r_{\mathrm{AIR}} x_{7} x_{3}+r_{\mathrm{Co}} x_{8}-k_{G} x_{3} \\
\dot{x}_{4}= & T_{l} x_{2}-x_{4} / H_{\mathrm{sp}}-k_{G} x_{4} \\
\dot{x}_{5}= & T_{l} x_{2}-x_{5} / H_{\mathrm{sp}}-k_{G} x_{5} \\
\dot{x}_{6}= & T_{l} x_{2}-x_{6} / H_{\mathrm{up}}-k_{G} x_{6} \\
\dot{x}_{7}= & x_{0}\left(r_{\mathrm{AII}} x_{4}-r_{\mathrm{AIR}} x_{7} x_{3}+r_{\mathrm{Co}} x_{8}\right)-x_{7} / H_{\mathrm{AI}} \\
\dot{x}_{8}= & r_{\mathrm{AIR}} x_{7} x_{3}-x_{8} / H_{\mathrm{sp}}-r_{\mathrm{Co}} x_{8}-k_{G} x_{8}
\end{aligned}
$$

where

$$
k_{G}=k_{g}\left(1-x_{0} / x_{0 \max }\right)
$$

and $x_{i}$ are non-negative real numbers:

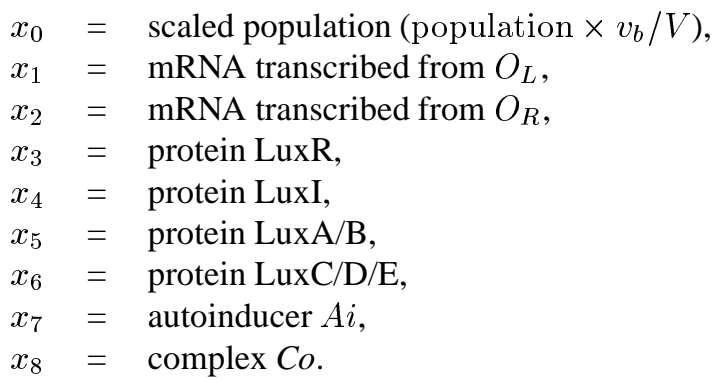

The parameters in model (3) are given in Table 1. The reader interested in their biological significance, units and estimated values is referred to [2]. $c_{\mathrm{CRP}}$ denotes the CRP concentration in the bacterium.

\subsection{Simplified hybrid model}

The continuous curve describing the regulation of transcription in Figure 2 is merely a convenient way of modeling the turning off of gene expression at low concentrations and the turning on of the gene at high concentrations. There is 


\begin{tabular}{|cl|}
\hline Parameter & Description \\
\hline$T_{c}$ & maximum transcription rate \\
$T_{l}$ & maximum translation rate \\
\hline$H_{\mathrm{RNA}}$ & RNA half life \\
$H_{\mathrm{sp}}$ & stable protein half-life \\
$H_{\mathrm{up}}$ & unstable protein half-life \\
$H_{\mathrm{AI}}$ & Ai half-life \\
\hline$r_{\mathrm{AII}}$ & rate const. at which LuxI makes $A i$ \\
$r_{\mathrm{AIR}}$ & rate const. $A i$ binding to LuxR \\
$r_{\mathrm{Co}}$ & rate const. of $C o$ dissociation \\
\hline$\nu_{\mathrm{CRPr}}$ & cooperativity coef. for CRP \\
$\kappa_{\mathrm{CRPr}}$ & half maximum conc. for CRP \\
$\nu_{\mathrm{Co}-\mathrm{icdabeg}}$ & cooperativity coef. for $C o$ \\
$\kappa_{\mathrm{Co}-\mathrm{icdabeg}}$ & half maximum conc. for $\mathrm{Co}$ \\
\hline$b$ & basal transcription rate \\
\hline$v_{b}$ & volume of a bacterium \\
$V$ & volume of solution \\
$k_{g}$ & growth rate \\
$x_{0 \mathrm{max}}$ & maximum population \\
\hline
\end{tabular}

Table 1: Model parameters - notation and description.

little experimental data confirming the exact shape of the sigmoid curve in the figure and we lack experimental data needed to estimate parameters like $\kappa$ and $\nu$, as in (1). In the absence of such data, it is simpler to pursue piece-wise constant approximations to the function that map the degree of activation/inhibition of transcription of a gene to intervals of concentration of activator/inhibitor. Figure 2 shows models that effectively give different levels of activation of a gene as a function of the concentration of the regulator. One can also imagine a more sophisticated $n$-step approximation. From a system analysis point of view, dealing with such piecewise constant functions describing transcription is advantageous, because it allows us to abstract a nonlinear system as a switched, lower dimensional, system that may be easier to analyze because of the absence of the sigmoid nonlinearity.

To derive the simplified hybrid model, we disregard the growth of the population $x_{0}$ and, consequently, the dilution. This situation corresponds to a completely grown or to a growth-controlled population. Also, the mRNA dynamics are assumed to be fast (instantaneous) [2]. With these assumptions, the equations for mRNA become linear and independent of the other equations in the model. Moreover, the mRNA concentrations can be assumed to be switching among constant levels, the switching being determined by the concentrations of their corresponding activators/inhibitors. This gives a partition of the state space into modes with specific dynamics. More specifically, we assume $m$ levels of activation of the luxICDABEG gene at $c_{0}, c_{1}, \ldots, c_{m-1}$ with $c_{0}=0$ and $c_{m-1}=1$. The level of activation of the luxICDABEG gene is $c_{i}$ when $x_{8 s w}^{i}<$ $x_{8}<x_{18 s w}^{i+1}$ where $x_{12 s w}^{0}=0$ and $x_{12 s w}^{m}=\infty$. Similarly, for the luxR gene, we assume $n$ steps at $d_{0}, d_{1}, \ldots, d_{n-1}$, $d_{0}=0, d_{n-1}=1$, and the activation is at level $d_{j}$ when
$c_{C R P s w}^{j}<c_{C R P}<c_{C R P s w}^{j+1}$. A generic mode labeled (ij) $(i=1, \ldots, m-1, j=1, \ldots, n-1)$ has constant mRNA concentrations given by:

$$
\begin{gathered}
x_{1}^{i j}=H_{R N A} T_{c}\left[\left(1-c_{i}\right) d_{j}+b\right], \\
x_{2}^{i j}=H_{R N A} T_{c}\left[c_{i}\left(1-d_{j}\right)+b\right]
\end{gathered}
$$

In (3, the non-trivial dynamics are described by

$$
\dot{x}=A x+g(x)+b_{i j}, \quad x=\left[\begin{array}{lll}
x_{3} & x_{4} x_{7} x_{8}
\end{array}\right]^{T}
$$

where

$$
\begin{gathered}
A=\left[\begin{array}{cccc}
-\frac{1}{H_{s p}} & 0 & 0 & r_{C o} \\
0 & -\frac{1}{H_{s p}} & 0 & 0 \\
0 & x_{0} r_{A I I} & -\frac{1}{H_{A I}} & x_{0} r_{C o} \\
0 & 0 & 0 & -\frac{1}{H_{s p}}-r_{C o}
\end{array}\right], \\
g(x)=r_{A I R} x_{7} x_{3} p
\end{gathered}
$$

$$
p=\left[\begin{array}{c}
-1 \\
0 \\
-x_{0} \\
1
\end{array}\right], \quad b_{i j}=\left[\begin{array}{c}
H_{R N A} T_{c} T_{l}\left[\left(1-c_{i}\right) d_{j}+b\right] \\
H_{R N A} T_{c} T_{l}\left[c_{i}\left(1-d_{j}\right)+b\right] \\
0 \\
0
\end{array}\right]
$$

and invariant sets given by

$$
x_{8 s w}^{i}<x_{8}<x_{8 s w}^{i+1} \bigwedge c_{C R P s w}^{j}<c_{C R P}<c_{C R P s w}^{j+1}
$$

\section{Simulation results}

Figure 3 (a) shows log-linear plots of numerical simulations of the continuous system in Section 2.1. for a constant $c_{\mathrm{CRP}}$ of $10 \mathrm{nM}$. The population (bottom) grows from $10^{5} / \mathrm{ml}$ to $10^{9} / \mathrm{ml}$ over about 15 hours then remains constant for an additional 20 hours. For all the species, there is a short initial adjustment period when protein, complex, and $A i$ concentrations reach a nearly constant state at low population density which lasts for roughly eight hours. LuxR is abundant, ready to sense increases in $A i$. On the other hand, $C o$, $L u x I, A i$, and total luminescence are low. As the population grows, the production of $A i$ begins to rise above the background, which triggers the formation of LuxR: $A i$ complex $C o$. Increasing $C o$ activates the positive feedback loop of $O_{R}$, LuxI, and $A i$. The concentrations of these components increase rapidly as does the total luminescence, and this is what one expects to get through experiments [4].

The simulation results of the switched model obtained from (3) by replacing the sigmoids with piecewise constant functions with three steps $(m=n=3)$ are shown in Figure 3 (b) for a constant CRP concentration of 10nM. The plots closely resemble the continuous simulations. The final equilibrium values are also in good agreement between the two models. 


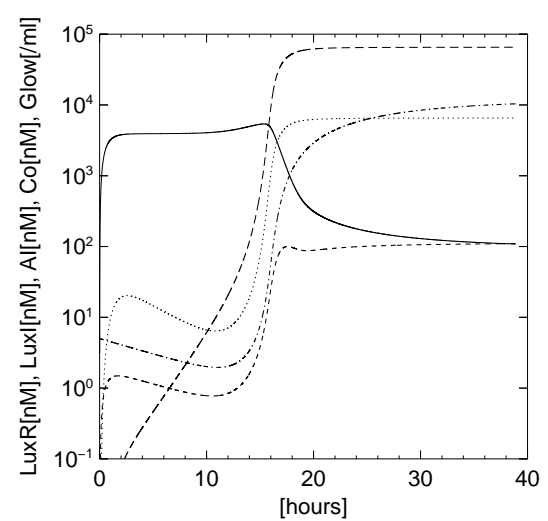

(a)

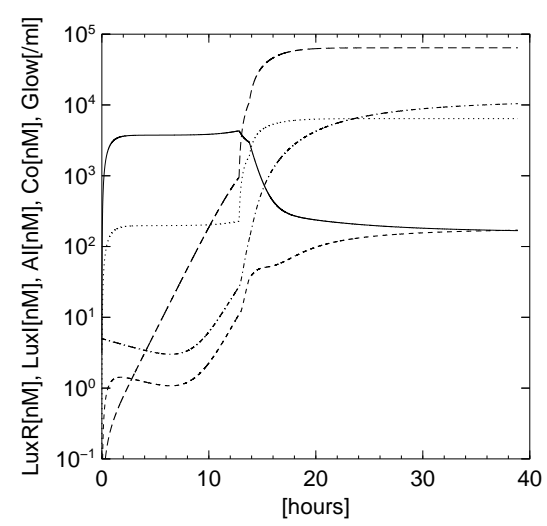

(b)

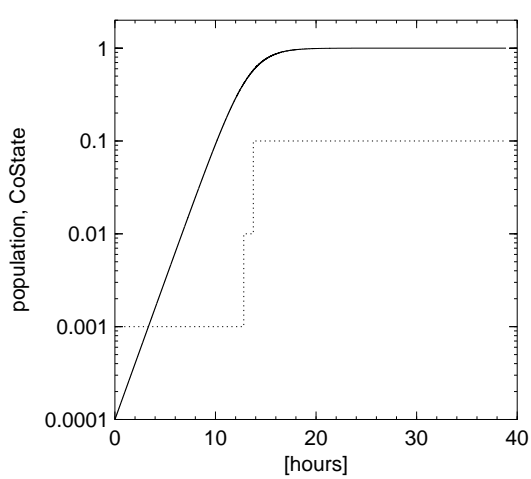

(c)

Figure 3: Time evolution of concentrations (in nM) during population growth: proteins LuxR (solid), LuxI (dotted), $A i$ (dot-dashed), $C o$ (dashed), and total luminescence (long dashes): (a)-continuous model, (b)-switched model. Population growth curve is shown in (c). The switch history for the hybrid model is shown dotted.

\section{Stability analysis}

For the simplified hybrid model in Section 2.2, equilibria and their stability are determined by the non-trivial dynamics in (4). The Jacobian $J_{i j}$ (whose expression is omitted for simplicity) of (4) at a feasible equilibrium $x_{e}^{i j}$ (i.e., all components are positive and the invariant (5) is satisfied) has two eigenvalues at $-1 / H_{s p}$ and the other two given by the roots of the polynomial

$$
\begin{gathered}
1+H_{s p} r_{C o}+H_{s p} r_{A I R} x_{e 7}^{i j}+x_{0} H_{A I} r_{A I R} x_{e 3}^{i j} \\
+\left(H_{A I}+H_{s p}+H_{A I} H_{s p} r_{C o}+H_{A I} H_{s p} r_{A I R} x_{e 7}^{i j}+\right. \\
\left.x_{0} H_{A I} H_{s p} r_{A I R} x_{e 3}^{i j}\right) \lambda+H_{A I} H_{s p} \lambda^{2}
\end{gathered}
$$

Since the coefficients of the above second order polynomial are strictly positive for all positive values of the constants, we conclude that, if a feasible equilibrium $x_{e}^{i j}$ for mode (ij) exists, then it is locally asymptotically stable, for all feasible values of the parameters.

An estimate of the region of attraction can be easily constructed around the (possible) equilibrium of each mode due to the special form of the nonlinearity. By abuse of notation, let the translated state $x-x_{e}^{i j}$ be denoted by $x$ again. The system describing the dynamics of mode (ij) around its equilibrium translated at the origin is of the form

$$
\dot{x}=J_{i j} x+g(x)
$$

where $J_{i j}$ is Hurwitz. We use the vanishing perturbation technique [6] to construct an ellipsoidal region of attraction around the origin of (6), and, eventually, translate the ellipsoid back around $x_{e}^{i j}$ in the original space. Let $P_{i j}$ be the unique symmetric, positive definite solution of the Lyapunov equation $P_{i j} J_{i j}+J_{i j}^{T} P_{i j}=-I$.

Let $V(x)=x^{T} P_{i j} x$ be the corresponding Lyapunov function for the linear part of (6) and a possible candidate for the nonlinear system. The derivative of $V(x)$ along the trajectories of (6) is given by:

$$
\begin{aligned}
& \dot{V}(x)=-\|x\|^{2}+2 x^{T} P_{i j} g(x) \\
& =-\|x\|^{2}+2 r_{A I R} x_{7} x_{3} x^{T} P_{i j} p
\end{aligned}
$$

If $x$ is in the region between two hyperplanes in $\mathbb{R}^{4}$ containing the origin of the form $\left|x^{T} P_{i j} p\right|<\kappa$, then it is easy to see that $\kappa<1 / r_{A I R}$ is a sufficient condition for $\dot{V}(x)<0$. To find an estimate for $\kappa$, we solved the constrained optimization problem

$$
\max _{x, x^{T} P_{i j} x=c}\left|x^{T} P_{i j} p\right|=\sqrt{c} \sqrt{p^{T} P_{i j} p}
$$

The conclusion is that the maximum ellipsoid of attraction is of the form

$$
V(x)=x^{T} P_{i j} x \leq \frac{1}{r_{A I R}^{2} p^{T} P_{i j} p}
$$

If the ellipsoid described by (7) is completely included in the invariant of mode (ij), then we can conclude that it is indeed an attractive set for the switched system (4). Otherwise, it needs to be shrunk until the inclusion condition is satisfied.

Besides stability, another interesting question for both biologists and control engineers would be what makes the system switch among modes. For example, given a (desired, luminescent) stable equilibrium, with the above method we can construct a region of attraction within the corresponding mode, but can the ellipsoid be reached from other (nonluminescent) modes? This is a reachability problem, which we cannot solve using existing tools. However, we can solve such reachability problems for a mutant of $V$. fischeri where luminescence is described by linear switched dynamics. 


\section{Reachability analysis of a mutant of $V$. fischeri}

Another interesting question from a biologist's standpoint relates to reachability. For example, can a cell population from a specified initial condition reach an equilibrium that corresponds to luminescence. Obviously the stability of the equilibrium does not help us answer this question. This is a so-called reachability problem. In this section, model a mutant of $V$. fischeri where the luminescence is described by linear switched dynamics, and show how such questions can be answered.

We consider the mutant of $V$. fischeri described in [9] that disrupts the operon structure, and, therefore, the regulatory system described above. In the mutant, the luxR gene is deleted from its normal location and a copy is placed in a plasmid (a separate piece of DNA) and incorporated back into V. fischeri. The plasmid copy of luxR is under control of a different promoter that yields a constant, high rate of transcription unaffected by any of the molecules described above. We assume a constant concentration of protein LuxR at $x_{3}=10000 \mathrm{nM}$, and the CRP concentration is low (regulation kept at $d_{0}=0$ ). The model becomes linear with nontrivial dynamics for mode (ij) in the form $\dot{x}=A x+b_{i j}$, where $x=\left[\begin{array}{lll}x_{4} & x_{7} & x_{8}\end{array}\right]^{T}$ and

$$
\begin{gathered}
A=\left[\begin{array}{ccc}
-\frac{1}{H_{s p}} & 0 & 0 \\
x_{0} r_{A I I} & -\frac{1}{H_{A I}}-x_{0} R r_{A I R} & x_{0} r_{C o} \\
0 & R r_{A I R} & -\frac{1}{H_{s p}}-r_{C o}
\end{array}\right], \\
b_{i j}=\left[\begin{array}{c}
H_{R N A} T_{l} T_{c}\left[c_{i}\left(1-d_{j}\right)+b\right] \\
0 \\
0
\end{array}\right]
\end{gathered}
$$

The unique equilibrium of mode (ij) is given by:

$$
\begin{aligned}
x_{e 4}^{i j} & =\left(b+c_{i}-c_{i} d_{j}\right) H_{R N A} H_{s p} T_{c} T_{l} \\
x_{e 7}^{i j} & =\frac{x_{0} H_{A I} r_{A I I}\left(1+H_{s p} r_{C o}\right)}{1+x_{0} H_{A I} R r_{A I R}+H_{s p} r_{C o}} x_{e 4}^{i j} \\
x_{e 8}^{i j} & =\frac{x_{0} H_{A I} H_{s p} R r_{A I I} r_{A I R}}{1+x_{0} H_{A I} R r_{A I R}+H_{s p} r_{C o}} x_{e 4}^{i j}
\end{aligned}
$$

The eigenvalues of the matrix $A$ show that the equilibrium points are asymptotically stable.

Note that the stability result is not a global result. The eigenvalue analysis tells us that if the system starts from a point within the mode (ij), it converges to the equilibrium point. However, it does not tell us anything about initial conditions that may lie in a different mode. This is the main motivation for reachability analysis.

We consider a simple case study in which the system is composed of two modes (00) and (10), which we call nonluminescent (non-lum) and luminescent (lum), respectively. For our estimated values of the parameters [2], the equilibria are given by: $x_{e 4}^{\text {non-lum }}=13.5, x_{e 7}^{\text {non-lum }}=36.18$, $x_{e 8}^{\text {non-lum }}=17.84$ and $x_{e 4}^{\text {lum }}=13513.5, x_{e 7}^{\text {lum }}=36218.5$, $x_{e 8}^{\operatorname{lum}}=17861.2$, respectively. Assuming that the luxICD$A B E G$ gene is switched on at $x_{8 s w}=1000 \mathrm{nM}$, both the above equilibria are feasible. An interesting question from a biological point of view would be under what conditions the system arrives at the luminescent equilibrium, and particularly, what makes the system switch and turn on the luxICDABEG gene?

To determine the states from which the system can reach the equilibrium $x_{e}^{\text {lum }}$, consider a small neighborhood $F$ around $x_{e}^{\text {lum }}$ and determine all states that can reach $F$ by performing backward reachability analysis. The two modes lead to the hybrid automaton model [1] shown in Figure 4). The

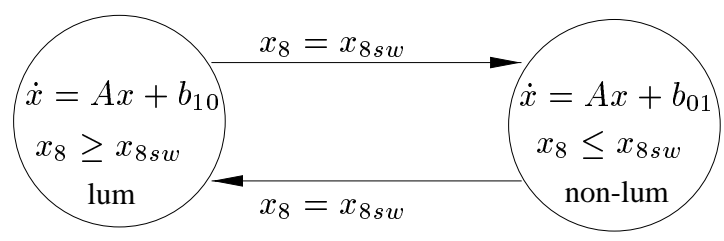

Figure 4: The hybrid automaton of the mutant system.

reachability computations are done using $d / d t$ [3]. Basically, $d / d t$ is a tool that uses polyhedra to over- or underapproximate reachable sets of hybrid automata. For our problem, an under-approximation is required since we need to make sure that all the states in the computed set go to the equilibrium $x_{e}^{\mathrm{lum}}$. In our case, we define the neighborhood $F$ as the box $[13500,13525] \times[36200.0,36230.0] \times$ $[17860,17868]$ and study the system's behavior within the set $[0,30000] \times[0,60000] \times[0,30000]$. The tool computes first the set $\mathcal{R}_{\text {lum }}$ of states backward reachable from $F$ by mode lum (see Figure 5 (a)). Then, to determine the states from which the system can switch to mode non-lum (backward in time), the intersection of $\mathcal{R}_{\text {lum }}$ with the switching plane $x_{8}=x_{8 s w}$ is computed, which gives the set $G=[0,30000] \times[1400.2,42926.5] \times[1000,1000]$. Starting from the intersection, the tool computes the set $\mathcal{R}_{\text {non-lum }}$ backward reachable by mode non-lum (see Figure 5 (b)). This example illustrates how one can predict the initial conditions from which luminescence can be achieved.

\section{Conclusion}

In this paper ${ }^{2}$ we presented tools that could be used to study stability and reachability properties of biological systems, with application to quorum sensing in the marine bacterium Vibrio fischeri. We showed by simulation that a growing population determines building of a small molecule called autoinducer $A i$ and, eventually, luminescence. We presented how a reduced, hybrid model of the regulatory network can be constructed and emphasized its usefulness. Using $d / d t$, a safety verification tool for hybrid linear sys-

\footnotetext{
${ }^{2}$ This paper is part of a large collaborative effort that includes Rajeev Alur, Franjo Ivancic, Insup Lee, and Max Mintz.
} 


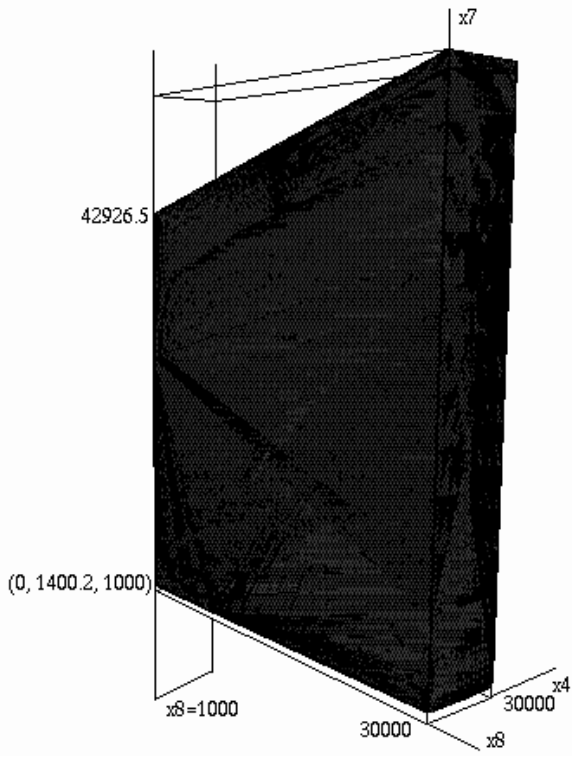

(a)

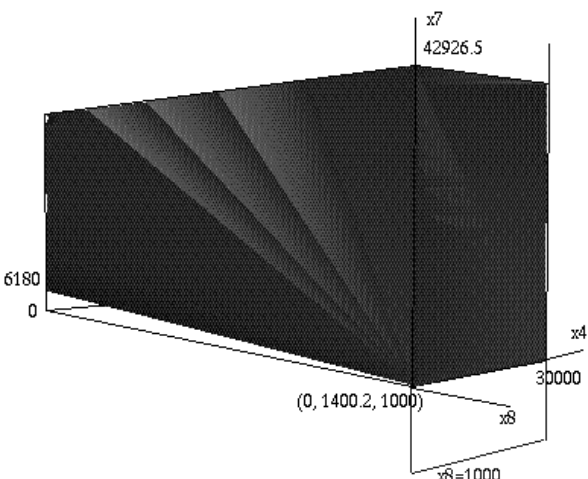

(b)

Figure 5: (a) The set $\mathcal{R}_{\text {lum }}$ of states backward reachable by mode lum (the box $F$ is hidden inside $\mathcal{R}_{\text {lum }}$ ); (b) The set $\mathcal{R}_{n o n-l u m}$ of states backward reachable by mode non-lum ( $G$ is the rectangle on the switching plane $x_{8}=1000$ ).

tems, we derived sufficient conditions for reachability of the stable luminescent state. While the results of the calculations performed in this paper are consistent with the experimental observations, it is premature to conclude that our models or the analysis provide a significantly new insight into the biology. The results however do point to a set of exciting directions for basic research in modeling and tool development which will eventually allow the in-silico design and analysis of biological networks, hypothesis generation, testing and evaluation thus complementing sophisticated but time consuming and expensive biological experiments. Our website http: / / www. cis . upenn. edu/mobies/charon/ provides additional information about our programming language CHARON and analysis tools for hybrid systems. More information is also available at http://www. cis. upenn. edu/biocomp/.

\section{References}

[1] R. Alur, C. Courcoubetis, N. Halbwachs, T.A. Henzinger, P.-H. Ho, X. Nicollin, A. Olivero, J. Sifakis, and $\mathrm{S}$. Yovine. The algorithmic analysis of hybrid systems. Theoretical Computer Science, 138:3-34, 1995.

[2] C. Belta, J. Schug, T. Dang, V. Kumar, G. J. Pappas, H. Rubin, and P. Dunlap. Stability and reachability analysis of a hybrid mnodel of luminescence in the marine bacterium V.fischeri. Grasp lab, University of Pennsylvania, Philadelphia, PA, 2001.

[3] T. Dang. Verification and Synthesis of Hybrid Systems. PhD thesis, Institut National Polytechnique de Grenoble, Laboratoire Verimag, 2000.
[4] P. V. Dunlap. Quorum regulation of luminescence in vibrio fischeri. In D. Bartlett, editor, Molecular marine microbiology, pages 3-21. Horizon Press, Norfolk, UK, 2000.

[5] R. Heinrich and S. Schuster. The regulation of cellular systems. International Thomson Publishing, New York, 1996.

[6] H. Khalil. Nonlinear Systems. Prentice Hall, Upper Sadle River, NJ, 2nd edition, 1996.

[7] N. Lynch and B. H. Krogh, editors. Hybrid Systems: Computation and Control, volume 1790 of LNCS. SpringerVerlag, 2000.

[8] S. Morse, editor. Control Using Logic-Based Switching, volume 222 of Lecture Notes in Control and information sciences. Springer-Verlag, 1997.

[9] P. V. Dunlap and A. Kuo. Cell density-dependent modulation of the Vibrio fischeri luminescence system in the absence of autoinducer and LuxR protein. J Bacteriol, 174(8):2440-8., 1992.

[10] D. M. Sitnikov, J. B. Schineller, and T. O. Baldwin. Transcriptional regulation of bioluminescence genes from vibrio fischeri. Molecular microbiology, 17(5):801-812, 1995.

[11] S. Swift, P. Williams, and G. S. A. B. Steward. Nacylhomoserine lactones and quorum sensing in proteobacteria. In G. Dunny and S. C. Winans, editors, Cell-cell signaling in bacteria, pages 291-313. American Society for Microbiology, Washington, DC, 1997. 